九州大学学術情報リポジトリ

Kyushu University Institutional Repository

\title{
STRUCTURE OF DETERMINATIVE SUBSPACE IN TRIANGULAR CELL SPACE : INFORMATION SCIENCE APPROACH TO BIOMATHEMATICS, VIII
}

Yamaguchi, Masako

Research Institute of Fundamental Information Science, Faculty of Science, Kyushu University

https://doi.org/10.5109/13056

出版情報: 統計数理研究. 14 (3/4)，pp. 107-124，1971-03. Research Association of Statistical Sciences バージョン：

権利関係 : 


\title{
STRUCTURE OF DETERMINATIVE SUBSPACE IN TRIANGULAR CELL SPACE \\ INFORMATION SCIENCE APPROACH TO BIOMATHEMATICS, VIII
}

\author{
Masako YAMAGUCHI*
}

(Received January 16, 1971)

\section{$\S 1$. Introduction}

The notion of stable configuration in a $J^{(n)}$ cell space which is invariant under any application of local majority transformation (LMT) was introduced in Kitagawa and Yamaguchi [1] and the notion of determinative subspace in a $\Delta^{(n)}$ cell space which determines a structure of a stable configuration was introduced in our paper [2]. Some structual properties of determinative subspace in $\Delta^{(n)}$ cell space were suggested throughout various examples of determinative subspace in Kitagawa and Yamaguchi [3]. According to the definitions of generative and non-generative determinative subspace in $A^{(n)}$ cell space given by Kitagawa [4] in view of propagation of determined cells, these examples are mostly concerned with those of generative determinative subspace in $J^{(n)}$ cell space.

The purpose of this paper is to give a deeper investigation for the constructions of determinative subspaces in $S^{(n)}$ cell space.

In Section 2 we shall introduce several notions which are indispensable for a construction procedure of generative determinative subspace in $\Delta^{(n)}$ cell space such as a convex set, a spiny convex set and a two-cell extension of a set and so on.

In SECTION 3 we shall give a certain type of construction procedure of any generative determinative subspace in $S^{(n)}$ cell space. In APPENDIX we shall give an example of our construction process of a generative determinative subspace obtained by using this proceduce.

In SECTION 4 we shall introduce several notions of elementary subsets and superposition of elementary subsets and decomposition of the whole cell space into a family of superposed elementary subsets. These nations are fundamental tools for proving THEOREM 3 and 4, which give us a construction procedure and hence a structural characteristic feature of any non-generative determinative subspace in $J^{(n)}$ cell space.

The last SECTION 5 is devoted to the another proof of THEOREM 2 in our previours paper [2] which appeals to LEMMA 4 in the present paper prepared for giving our construction procedure of non-generative determinative determinative

* Research Institute of the Fundamental Information Science, Faculty of Science, Kyushu University, Fukuoka. 
subspace in the $A^{(n)}$ cell space.

\section{§. Preparatory consideration and definitions}

The notion of determinative subspace in $A^{(n)}$ cell space was introduced by Yamaguchi [2]. For the sake of completeness, let us give here its definition.

Definition 1. A subset D of cells in $S^{(n)}$ cell space is said to be a determinative (cell) subspace in $S^{(n)}$ cell space if it satisfies the following two conditions:

(1) To any assigned configuration in the set D there corresponds one and only one stable configuration in $J^{(n)}$ cell space whose restriction in the set $\mathrm{D}$ is conincident with the given configuration in the set $D$.

(2) Any stable configuration in the $J^{(n)}$ cell space is uniquely determined by its restriction in the set $D$.

The notions of generative and non-generative determinative subspace given by Kitagawa [3] may be described by the following two definitions respectively.

Definition 2. A determinative subspace $\mathrm{D}$ in $A^{(n)}$ cell space is said to be a generative determinative subspace, abbreviated by GDS, if a determination of a stable configuration in $J^{(n)}$ cell space is performed step by step in a sequential way by $D$.

Let us call a process of such a determination by a propagation and call a $J^{(2)}$ basic space determined by the first propagation by a source. Of cource, such a $\Delta^{(2)}$ basic space has to contain three cells in GDS in order to be a source and for any GDS in a $J^{(n)}$ cell space may have more than one source. (see EXAMPLE in APPENDIX)

Definition 3. A determinative subspace in $S^{(n)}$ cell space is said to be a nongenerative determinative subspace, abbreciated by NGDS, if it is not a GDS.

Examples of GDS and NGDS are given in Figure 2.1, (a) and (b), respectively. In Figure 2.1, (a) the GDS consists of the following cells:

$$
\begin{array}{lll}
\left(1^{\circ}\right) & (1, j) & (j=1 \sim 19) \\
\left(2^{\circ}\right) & (i, 2) & (i=2 \sim 9) .
\end{array}
$$

The sole source of GDS is the $J^{(2)}$ basic space consisting of the set cells $\{(1,1)$, $(1,2),(1,3),(2,1)\}$. In Figure 2.1 , (b) consists of the following cells :

$$
\begin{array}{lll}
\left(1^{\circ}\right) & (1, j) & (j=1 \sim 8,10 \sim 19) \\
\left(2^{\circ}\right) & (i, 2) & (i=2 \sim 9) \\
\left(3^{\circ}\right) & (4,7) &
\end{array}
$$

The fact that the subspace (2.1) is a GDS is given by the proof of THEOREM 1 in our previous paper [3]. The proof for the assertion that the subspace (2.2) is a NGDS will be given by THEOREM 2 in SECTION 4 of this paper.

For the sake of convenience we shall adopt

DEFINITION 4. A subset $\mathrm{T}$ in a $\Delta^{(n)}$ cell space is said to be determined by a subset $S$ of the space if a state of each cell in $T$ is determined in a unique way for any assignment of configurations in the set $S$.

Definition 5. Two cells $(i, j)$ and $\left(i^{\prime}, j^{\prime}\right)$ in $S^{(n)}$ cell space are said to belong to 


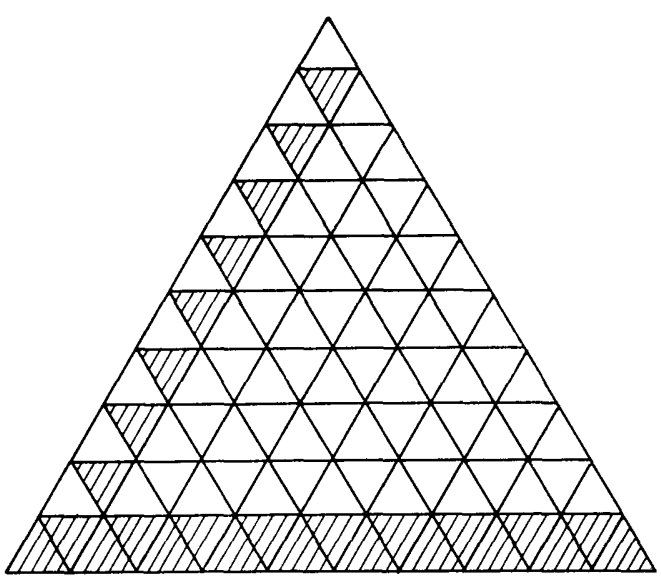

Fig. 2.1, (a). GDS in $\Delta^{(10)}$ cell space.

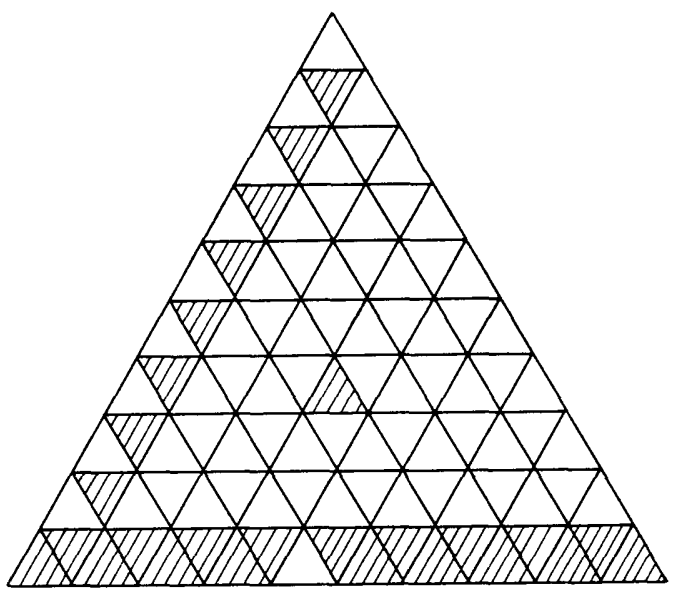

Fig. 2.1, (b). NGDS in $\Delta^{(10)}$ cell space.

the same generation with reference to a subset $S$ of a GDS if they satisfy the following two conditions:

(1) The set $\mathrm{S}+(i, j)$ or $\mathrm{S}+\left(i^{\prime}, j^{\prime}\right)$ is the subset of the GDS.

(2) The cell $\left(i^{\prime}, j^{\prime}\right)$ is determined by the set $\mathrm{S}+(i, j)$ and the cell $(i, j)$ is determined by the set $\mathrm{S}+\left(i^{\prime}, j^{\prime}\right)$.

Each determination in (2) is unique according to the Definition 4. For a subset $\mathrm{S}$ of a GDS we shall denote by $\mathrm{G}_{\mathrm{S}}(i, i)$ the set of all cells each of which belongs to the same generation with a cell $(i, j)$ with reference to $\mathrm{S}$.

DEFINITION 6. Two cells $c_{1}$ and $c_{2}$ are said to be adjacent if they have one common side.

DEFINITION 7. A set of cells in $\Delta^{(n)}$ cell space is said to be a path between $c_{1}$ and $c_{2}$ when there exists a sequence of cells $\left\{c_{i_{j}} ; j=1,2, \cdots, m\right\}$ such that

(1) $c_{i_{1}}=c_{1}$

(2) $c_{i_{m}}=c_{2}$

(3) $c_{i_{j}}$ and $c_{i_{j+1}}$ are adjacent cells for $j=1,2, \cdots, m-1$ respectively.

The number $m$ is called the length of the path. A set $\mathrm{S}$ of $\Delta^{(n)}$ is said to contain the path if all the cells $\left\{c_{i j}\right\}$ belong to $\mathrm{S}$.

DEFinition 8. A set $\mathrm{S}$ in $\Delta^{(n)}$ cell space is said to be convex if, for any assigned two cells $c_{1}$ and $c_{2}$ belonging to $S$, the set $S$ contain all the possible shortest paths between $c_{1}$ and $c_{2}$ in $\Delta^{(n)}$.

LEMMA 1. A convex set in a $S^{(n)}$ cell space is one of the polygons of the following five types:

(1) triangle;

(2) parallel quadrangle;

(3) trapezoed;

(4) pentagon;

(5) hexagon.

Proof. Any side of set $\mathrm{S}$ is parallel to one of the three sides of $\Delta^{(n)}$ cell space. Let us designate three directions of these sides by $a, b$ and $c$. Consequently any polygon in $\Delta^{(n)}$ cell space can be denoted by the ordered sequence of these three directions such $a b c$ and $a b a b$ and so on. Now in order that the set is convex, there exist several restrictions on the sequences of these directions. By a direct 
enumeration proceduce it is easy to show that there are substantially five types of sequences it is easy to show that there are substantially five types of sequences of these directions
(1) $a b c$
(2) $a b a b$
(3) $a b a c$
(4) $a b a c b$
(5) $a c b c b$,

which gives us the proof of our LEMMA.

EXAMPLE. Five types of convex polygons are illustrated in the following Figure 2.2, (1) (5).

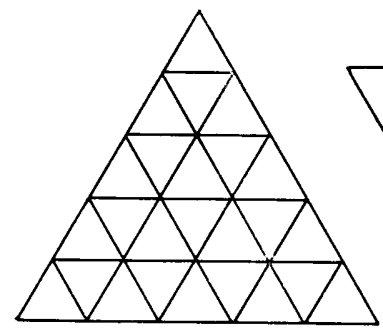

Fig. 2.2, (1). Convex set of triangle type.

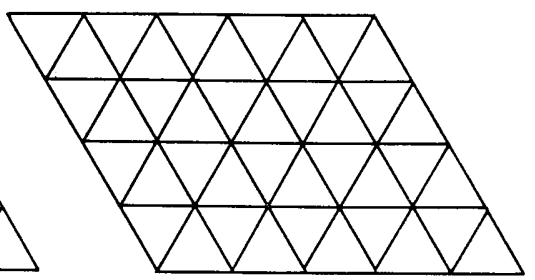

Fig. 2.2, (2). Convex set cf parallel quadrangle type.

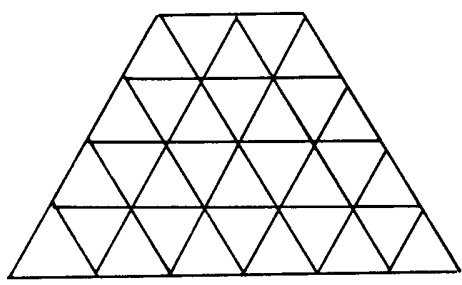

Fig. 2.2, (3). Convex set of trapezoid type.

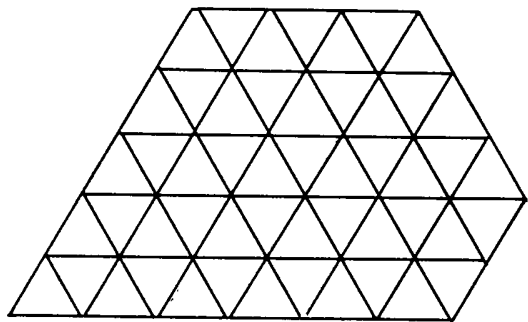

Fig. 2.2, (4). Convex set of pentagon type.

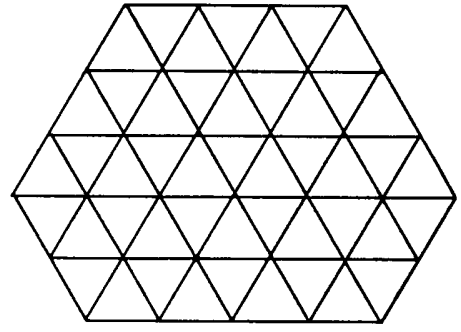

Fig. 2.2, (5). Convex set of hexagon type.

Definition 9. A set $\widetilde{\mathrm{S}}$ of cells in a $\Delta^{(n)}$ cell space is said to be a spiny convex set in $\Delta^{(n)}$ cell space if there exists a convex set $\mathrm{S}$ such that the set $\widetilde{\mathrm{S}}$ consists of the set $S$ and the set of all cells which belong to the space and are adjacent to the cells in the boundary of the convex set S.

EXAmple. Let $\mathrm{S}$ be a convex pentagon in a $\Delta^{(n)}$ cell space. Three examples of $\widetilde{\mathrm{S}}$ are given in Figure 2.3, in order to show the difference due to its location in the $\Delta^{(n)}$ space.

Definition 10. A set $\mathrm{S}^{*}$ of cells in a $\Delta^{(n)}$ cell space is said to be a two-cell extension of a set $\mathrm{S}$ in a $\Delta^{(n)}$ cell space if $\mathrm{S}^{*}$ consists of all cells in the space each of which there exists at least one a $\Delta^{(2)}$ basic space containing the cell and at least two other cells belonging to the set $\mathrm{S}$.

Example. Let $\mathrm{S}$ be a pentagon given in Figure 2.4. Then the two-cell extension $\mathrm{S}^{*}$ of $\mathrm{S}$ is given by the sum of two sets $\mathrm{S}$ and the set of cells with hatches in Figure 2.4 . 


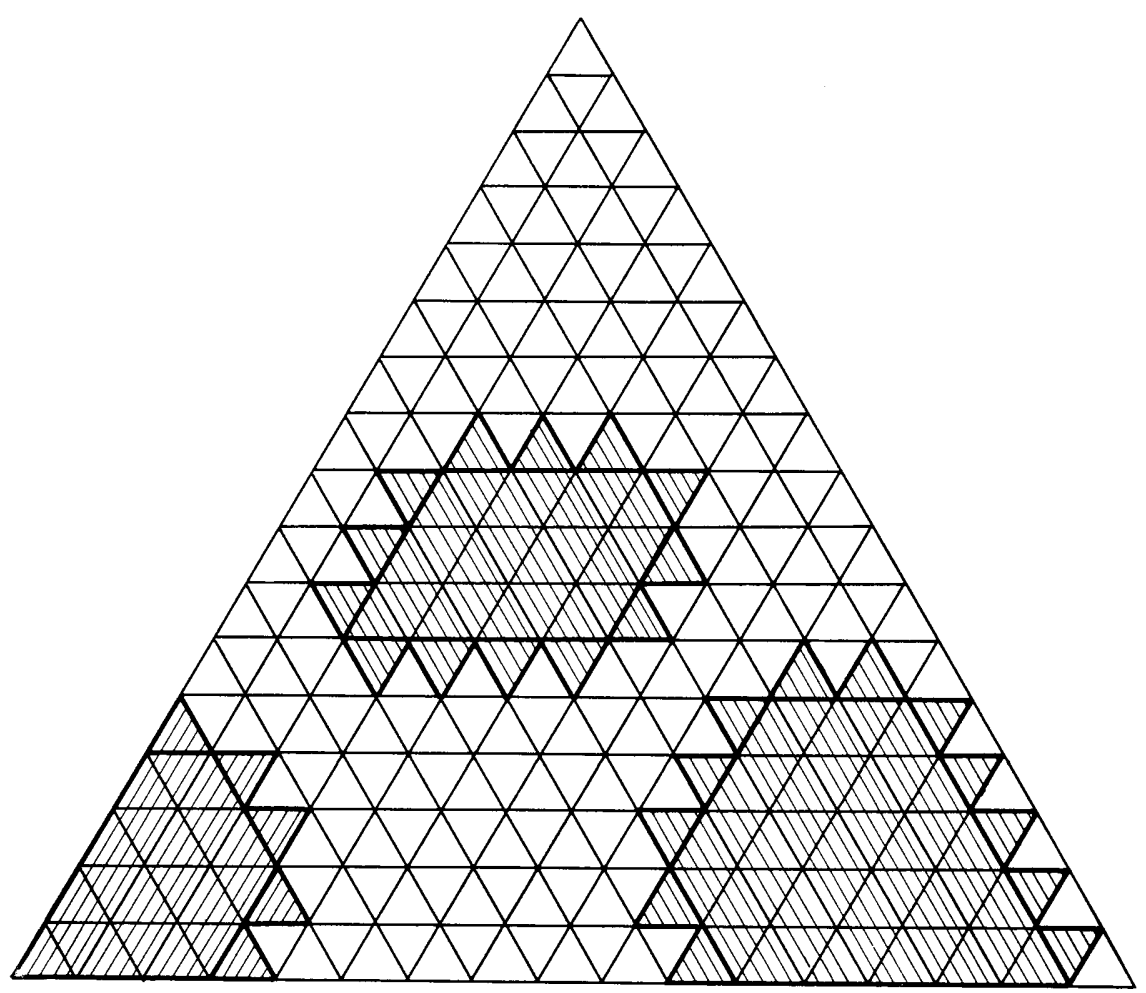

Fig. 2.3. Three examples of spiny convex set $\tilde{\mathrm{S}}$ with the convex pentagon.

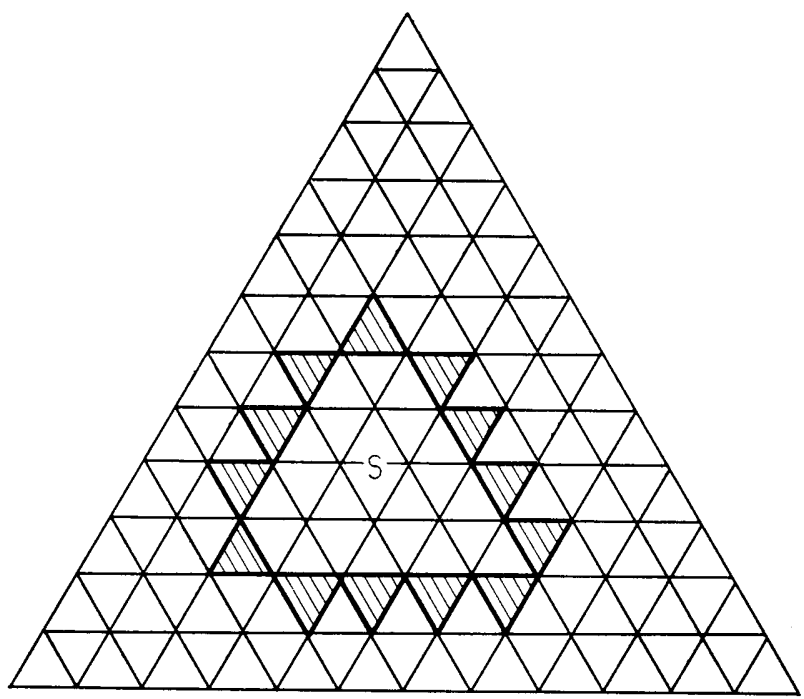

Fig. 2.4. Two-cell extension $\mathrm{S}^{*}$ of convex pentagon S. 
EXAMPLE. Let $\mathrm{S}$ be a spiny trapezoid shown in Figure 2.5. Then the two-cell extension $S^{*}$ of $S$ is given by the sum of two sets $S$ and the set of cells with hatches in Figure 2.5.

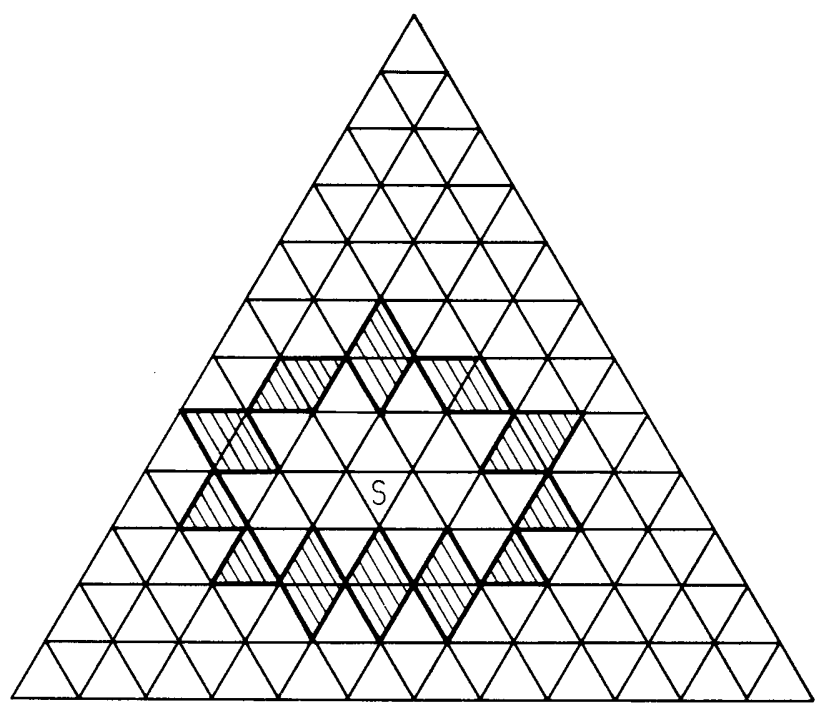

Fig. 2.5. Two-cell extension $\mathrm{S}^{*}$ of spiny trapezoid $\mathrm{S}$.

\section{$\S 3$. Construction of generative determinative subspaces in $A^{(n)}$ cell space}

Let us now introduce a certain type of sequential way of construction of a GDS in a $f^{(n)}$ cell space starting with a single source of propagation, which is given by the following.

DEFINITION 11. A procedure is said to be a successive cell adding procedure using two-cell extension sequence, if it is defined successively by the following steps :

STEP (1) Let us choose an arbitrary $\Delta^{(2)}$ basie space in the $\Delta^{(n)}$ cell space as the source of propagation. Now let us choose three arbitrary cells contained in the $\Delta^{(2)}$ basic space as the cells belonging to the GDS so that the remaining one cell is determined by these cells in order to be stable. Let us denote by $D_{1}$ the set of these choosen three cells and by $S_{1}$ the subset whose configuration is uniquely determined by $\mathrm{D}_{1}$ (see Figure 3.1).

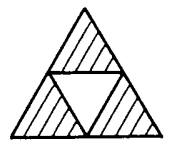

$D_{1}$

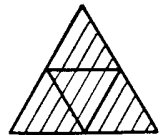

$\mathrm{S}_{1}$

Fig. 3.1.

STEP (2) At the second step the procedure deponds on the location of source whether it is on the couner or not (that is, either in the interior or on the boundary) 
in the $\Delta^{(n)}$ cell space. The construction procedure for each of these two cases is given in the following $\left(1^{\circ}\right)$ and $\left(2^{\circ}\right)$ respectively.

$\left(1^{\circ}\right)$ THE CASE OF SOURCE LOCATED EITHER IN THE INTERIOR OR ON THE BOUNDARY IN $\Delta^{(n)}$ CELL SPACE

Let us denote by $\mathrm{S}_{1}^{*}$ the two-cell extension $\mathrm{S}_{1}$ (see Figure 3.2, (a) and (b)).

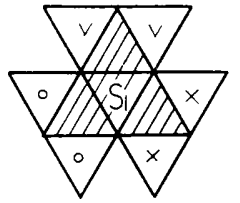

Fig. 3.2, (a). S* for the case of source located in the interion in $J^{(n)}$ cell space.

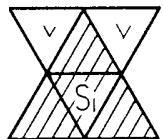

Fig. 3.2, (b). $\mathrm{S}_{1}^{*}$ for the case of source located on the boundary in $S^{(n)}$ cell space.

In Figure 3.2, (a) and (b) each set of two cells with the same mark (,$\times$ and $\vee$ ) belongs to the same generation with reference to $D_{1}$. Let us choose one arbitrary cell from $\mathrm{S}_{1}^{*}-\mathrm{S}_{1}$. Then the remaining one cell of the same generation is determined by the set consisting of $D_{1}$ and the choosen cell. Let us denote by $D_{2}$ the set adding the choosen cell to $D_{1}$ and by $S_{2}$ the set of cells determined by $D_{2}$ respectively.

STEP (3) Let us denote by $\mathrm{S}_{2}^{*}$ the two-cell extension of $\mathrm{S}_{2}$ (see Figure 3.3, (a) and (b)).

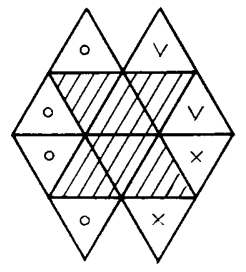

Fig. 3.3, (a). $\mathrm{S}_{3}^{*}$ for the case of source located in the interion in $J^{(n)}$ cell space.

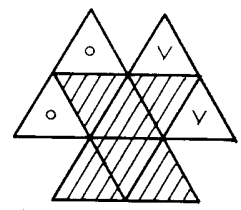

Fig. 3.3, (b). $\mathrm{S}_{2}^{*}$ for the case of source located on the boundary in $S^{(n)}$ cell space.

In Figure 3.3, (a) and (b) each pair of two cells with the same mark belongs to the same generation with reference to $D_{2}$. Let us choose one arbitrary cell from $\mathrm{S}_{2}^{*}-\mathrm{S}_{2}$. Then the romaining one cell with the same generation is determined by the set consisting of the choosen cell and $D_{2}$. Let us denote by $D_{3}$ the set adding

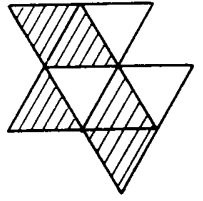

$D_{3}$

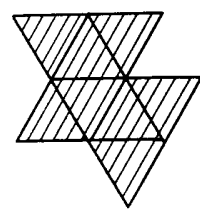

$\mathrm{S}_{3}$

Fig. 3.4. $D_{3}$ and $S_{3}$ for the case of source located in the interior in $\Delta^{(n)}$ cell space. 
the choosen cell to $\mathrm{D}_{2}$ and by $\mathrm{S}_{3}$ the set of cells determined by $\mathrm{D}_{3}$ respectively (see Figure 3.4, (a) and (b)).

STEP (i) Let us denote by $S_{i-1}^{*}$ the two-cell extension of $S_{i-1}$. Let us choose one arbitrary cell from $\mathrm{S}_{i-1}^{*}-\mathrm{S}_{i-1}$. Let us denote by $\mathrm{D}_{i}$ the set adding the choosen cell to $\mathrm{D}_{i-1}$ and by $\mathrm{S}_{i}$ the set of cells determined by $\mathrm{D}_{i}(i=3,4, \cdots)$.

$\left(2^{\circ}\right)$ The Case of source located in the comer in $\Delta^{(n)}$ CELl space

In this case there does not exist any two-cell extension of $S_{1}$. Therefore let us consider two $J^{(2)}$ basic spaces each of which contains one cell in $D_{1}$ (see Figure 3.5 ).

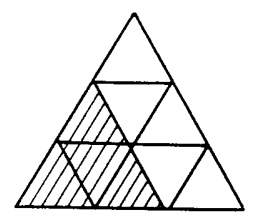

Fig. 3.5. The case of source located in the corner in $\Delta^{(n)}$ cell space.

Without loss of generality, let us consider the corner cell $(1,1)$. These are given by

$$
\begin{array}{llll}
{J_{1}^{(2)}}^{(2)}(\underline{\underline{(1,3)}} & (1,4) & (1,5) & (2,3) \\
{J_{2}^{(2)}}^{(2)} \underline{(2,1)} & (2,2) & (2,3) & (3,1)
\end{array}
$$

respectively, where the cells $(1,3)$ and $(2,1)$ belong to the source. Let us choose arbitharily one of these two basic spaces $\Delta_{1}^{(2)}$ and $\Delta_{2}^{(2)}$. From the choosen basic space, let us choose two cells which does not belong to the source. Let us denote by $\mathrm{D}_{2}$ the set adding these two cells to $D_{1}$ and by $S_{2}$ the set of cells determined by $D_{2}$. Except this initial construction of $\mathrm{D}_{2}$ and $\mathrm{S}_{2}$, our procedure in the case $\left(2^{\circ}\right)$ is quite similar to that of the case $\left(1^{\circ}\right)$ for the third step onward.

In connection with this procedure let us prepare with two Lemmas.

LEMMA 2. Each set $\mathrm{S}_{i}$ is a spiny convex set for $i=1,2,3, \cdots$.

Proof. Let us prove by induction. For the case $i=1$ and 2, Figures 3.1 and 3.3 , (a) or (b) give us a direct verification of the assertion of LEMMA 2. Now let us assume that each $S_{i}$ is a spiny convex set for $i=j$. We shall prove $S_{j+1}$ is a spiny convex set.

According to our definition of spiny convex set, there does exist a convex palygon $\mathrm{T}_{j}$ for the set $\mathrm{S}_{j}$. Since $\mathrm{T}_{j}$ is either of polygons of five types given in LEMMA 1 , it is evident that spines are given in the periphy of the convex polygon $T_{j}$. A cell chosen from the set $\mathrm{S}_{j}^{*}-\mathrm{S}_{j}$ induces a set of cells belonging to the same generation which is along the set of spines on the same side of the convex set $T_{j}$ as shown in Figure 3.6, (a) and (b), where the spine cells are devoted by the hatches.

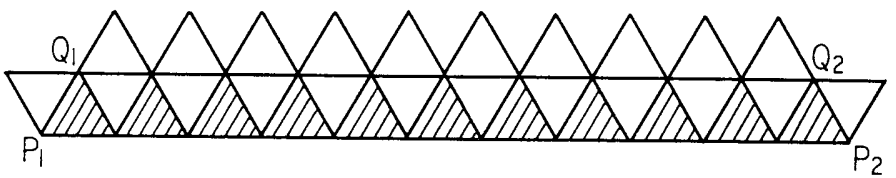

Fig. 3.6, (a).

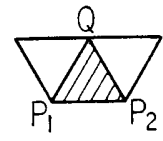

Fig. 3.6, (b).

It is to be noted that there does not happen any change along the other sides 
of the convex set $T_{j}$. In view of this process of cell adding and induced additional generation, it is easy to observe that the new convex set is now formed by the old sides in the convex set $T$ plus the line segments $P_{1} Q_{1} Q_{2} P_{2}$ and minus $P_{1} P_{2}$ for the case given in Figure 3.6, (a) and plus $\mathrm{P}_{1} \mathrm{QP}_{2}$ and minus $\mathrm{P}_{1} \mathrm{P}_{2}$ for the case given in Figure 3.6, (b). In either and both of these cases it is evident that we have obtained a convex set $T_{j+1}$ and $S_{j+1}$ is a spiny convex set, which completes the proof of our LEMMA 2.

LEMMA 3. If $\mathrm{S}_{i}$ is not coincident with the whole $\Delta^{(n)}$ space, there exists a $\mathrm{S}_{i+1}$ which is obtained by our cell-adding procedures and which is properly larger than $\mathrm{S}_{i}$.

PROOF. In view of LEMMA 1 and the proof of LEMMA 2, it turns out that, if $\mathrm{S}_{i}$ is not coincident with the whole $\Delta^{(n)}$ cell space, there exists at least one side or one corner for which at least one new cell can be added by our cell-adding procedure.

After these preparations we shall obtain

THEOREM 1. Any generative determinative subspace (GDS) in a $\Delta^{(n)}$ cell space can be understood as being given by a successive adding procedure using two-cell extension sequence given in DEFINITION 10.

Proof. Let D be a GDS in a $\Delta^{(n)}$ cell space. According to its definition, there does exist a source which is a $\Delta^{(2)}$ basic space whose three cells belong to the set D. The extension from the source to the whole space $\Delta^{(n)}$ can be understood by adding one cell in the set $\mathrm{D}$, irrespective of the situation whether they are given in the beginning or step by step as we have explained in our procedure given in DEFINITION 11. This implies that any denerative determinative subspace can be understood as being assigned according to the procedure given in DEFINITION 11. Now our LEMMA 2 and 3 it is evident that, so far as the construction can be understood as being given by our process, the extension from the source to the whole completes the proof of our THEOREM 1.

Let us give an example of our construction process of GDS in APPENDIX.

\section{$\S 4$. Construction of non-generative determinative subspace in $A^{(n)}$ cell space}

[1] Elementary subset Let us define the following notion which is indispensable to our construction procedure of NGDS.

Definition 12. For any assigned determinative subspace $D$ and for any assigned cell $(k, l)$ in $\mathrm{D}$, an $(k, l)$ elementary subset $\mathrm{E}_{D}(k, l)$ of $J^{(n)}$ cell space with respect to $\mathrm{D}$ is defined by the characteristic set function induced by the stable configuration $\left\{X_{i j}\right\}$ determined by the configuration $\mathrm{D}(X)$ in $\mathrm{D}$ such that $\mathrm{D}(X ;(k, l))=(0,0, \cdots$, $0,1,0, \cdots, 0)$, that is,

$$
\begin{array}{lll}
(i, j) \in \mathrm{E}_{D}(k, l) & \text { if } & x_{i j}=1 \\
(i, j) \notin \mathrm{E}_{D}(k, l) & \text { if } & x_{i j}=0 .
\end{array}
$$

A certain example of elementary subsets of $\Delta^{(n)}$ cell space were given in our paper Yamaguchi [2], where we used the notation $\left\{\mathrm{E}_{0}^{(n)}(k, l) ;(k, l) \in \mathrm{D}_{0}^{(n)}\right\}$, which is a family of the $(k, l)$ elementary subsets of $\Delta^{(n)}$ cell space with respect to the gen- 
erative determinative subspace $\mathrm{D}_{0}^{(n)}$ which is defined by the set of cells:

$(1, l)(1 \leqq l \leqq 2 n-1)$, and $(k, 2)(k=2,3, \cdots, n-1)$. This family of elementary subsets is particularly important for our consideration in what follows. For any assigned configuration $\mathrm{D}_{0}^{(n)}(X)=\left(\begin{array}{l}x_{11}, x_{12}, \cdots, x_{1,2 n-1} \\ x_{22}, x_{32}, \cdots, x_{n-1,2}\end{array}\right)$ in $\mathrm{D}_{0}^{(n)}$, a stable configuration in the whole $\Delta^{(n)}$ space is determined by $\mathrm{D}_{0}^{(n)}(X)$ and will be denoted by $\mathrm{C}(X)$. For any assigned cell $(k, l)$ in $\mathrm{D}_{0}^{(n)}$ and any assigned configuration $\mathrm{D}_{0}^{(n)}(X)$, the configuration $\mathrm{D}_{0}^{(n)}\left(X ; \overline{x_{k, l}}\right)$, which is defined as being identical with the configuration $\mathrm{D}_{0}^{(n)}(X)$ except for the cell $(k, l)$ where the state $x_{k, l}$ is now changed into $\overline{x_{k, l}}$, determines the stable configuration, which will be denoted by $\mathrm{C}_{(k, l)}(X)$.

Before we shall give a certain construction procedure to NGDS, let us prepare ourselves with the following fundamental

LEMMA 4. For any cell $(k, l)$ in $\mathrm{D}_{0}^{(n)}$, we have

$$
\mathrm{C}_{(k, l)}(X)=\mathrm{C}(X)+\mathrm{C}\left(\mathrm{E}_{0}^{(n)}(k, l)\right) .
$$

Proof. Because a cell $(k, l)$ belongs to $\mathrm{D}_{0}^{(n)}$, it suffices to consider the following two sets of cells $(1, l)(l=1,2, \cdots, 2 n-1)$ and $(k, 2)(k=2,3, \cdots, n-1)$.

Let us give the proof of (4.1) by a direct construction of stable configuration $\mathrm{C}_{(k, l)}(X)$. Now let us denote the state of $(i, j)$ cell in $\mathrm{C}(X)$ and $\mathrm{C}_{(k, l)}(X)$ by $x_{i j}$ and $y_{i j}$ respectively. (4.1) is equivalent to the relation

$$
y_{i, j}= \begin{cases}x_{i, j} & \text { if }(i, j) \oplus \mathrm{E}_{0}^{(n)}(k, l) \\ \overline{x_{i, j}} & \text { if }(i, j) \in \mathrm{E}_{0}^{(n)}(k, l)\end{cases}
$$

for any $(i, j)$ in $\Delta^{(n)}$ cell space.

In the $r$-th row of $\Delta^{(n)}$ cell space the state of $(r, j)$ cell is determined by the basic space $\{(r-1, j),(r-1, j+1),(r-1, j+2),(r, j)\}$ for an odd integer $j$ and by the basic space $\{(r-1, j),(r, j-2),(r, j-1),(r, j)\}$ for an even interg $j$, that is,

$$
\begin{array}{ll}
y_{r, j} \equiv y_{r-1, j}+y_{r-1, j+1}+y_{r-1, j+2}(\bmod 2) & \text { for } j=1,3, \cdots, 2(n-r)+1, \\
y_{r, j} \equiv y_{r-1, j}+y_{r, j-2}+y_{r, j-1}(\bmod 2) & \text { for } j=2,4, \cdots, 2(n-r) .
\end{array}
$$

In order to prove (4.2), let us appeal to mathematical induction. Let us first try the proof for any odd integer $l$.

(i) In the second row of $A^{(n)}$ cell space the state of $(2, j)$ for any odd interg $j$ is determined by (4.3) as the following relations

$$
\begin{aligned}
& y_{2, j} \equiv x_{1, j}+x_{1, j+1}+x_{1, j+2}(\bmod 2) \quad \text { for } j \neq l-2, l, \\
& y_{2, l-2} \equiv x_{1, l-2}+x_{1, l-1}+\overline{x_{1, l}}(\bmod 2), \\
& y_{2, l} \equiv \overline{x_{1, l}}+x_{1, l+1}+x_{1, l+2}(\bmod 2),
\end{aligned}
$$

which implies

$$
y_{2, j}= \begin{cases}\overline{x_{2, j}} & \text { if } j=l-2, l \\ x_{2, j} & \text { otherwise }\end{cases}
$$

for $j=1,3,5, \cdots, 2 n-3$. 
Now the state of $(2, j)$ cell for any even integer $j$ is determined by (4.4) as the following relation

$$
y_{2, j} \equiv x_{1, j}+y_{2, j-2}^{\prime}+y_{2, j-1}(\bmod 2)
$$

for $j=2,4, \cdots, 2 n-2$, which implies in view of (4.5) and $y_{2,2}=x_{2,2}$,

$$
y_{2, j}= \begin{cases}\overline{x_{2, j}} & \text { if } j=l \\ x_{2, j} & \text { otherwise }\end{cases}
$$

for $j=2,4, \cdots, 2 n-2$.

But, from (4.5) and (4.6) we have

$$
y_{2, j}= \begin{cases}\overline{x_{2, j}} & \text { if } j=l-1, l, l+1 \\ x_{2, j} & \text { otherwise, }\end{cases}
$$

which amounts to say that the assertion of LEMMA 4 is valid for the second row of $S^{(n)}$ cell space.

(ii) Assuming that the state of each cell $(r-1, j)$ in the $(r-1)$-th row of $4^{(n)}$ cell space is given by (4.2), let us prove that (4.2) holds for the $r$-th row $(1 \leqq r$ $\leqq(l-1) / 2)$. The state of $(r, j)$ cell for an odd integer $j$ is determined by (4.3) as the following relations

$$
\begin{array}{ll}
y_{r, j} \equiv x_{r-1, j}+x_{r-1, j+1}+x_{r-1, j+2}(\bmod 2) & \text { for } j \neq l-2(r-1), \cdots, l-2, l, \\
y_{r, j} \equiv \overline{x_{r-1, j}}+\overline{x_{r-1, j+1}}+\overline{x_{r-1, j \div 2}}(\bmod 2) & \text { for } j=l-2(r-2), \cdots, l-2, \\
y_{r, j} \equiv x_{r-1, j}+x_{r-1, j+1}+\overline{x_{r-1, j+2}}(\bmod 2) & \text { for } j=l-2(r-1), \\
y_{r, j} \equiv \overline{x_{r-1, j}}+x_{r-1, j+1}+x_{r-1, j+2}(\bmod 2) & \text { for } j=l,
\end{array}
$$

which implies

$$
y_{r, j}= \begin{cases}\overline{x_{r, j}} & \text { if } j=l-2(r-1), \cdots, l-2, l \\ x_{r, j} & \text { otherwise. }\end{cases}
$$

The state of $(r, j)$ cell for any even integer $j$ is given by (4.4), (4.7) and $y_{r, 2}=x_{r, 2}$ as the following relations

$$
\begin{array}{ll}
y_{r, j} \equiv x_{r-1, j}+x_{r, j-2}+x_{r, j-1}(\bmod 2) & \text { for } j=4,6, \cdots, l-2(r-1)-1, l+3, \cdots, 2(n-r), \\
y_{r, j} \equiv \overline{x_{r-1, j}}+\overline{x_{r, j-2}}+\overline{x_{r, j-1}}(\bmod 2) & \text { for } j=l-2(r-1)+3, \cdots, l-3, l-1, \\
y_{r, j} \equiv x_{r-1, j}+x_{r, j-2}+\overline{x_{r, j-1}}(\bmod 3) & \text { for } j=l-2(r-1)+1, \\
y_{r, j} \equiv x_{r-1, j}+\overline{x_{r, j-2}}+\overline{x_{r, j-1}}(\bmod 2) & \text { for } j=l+1,
\end{array}
$$

which implies

$$
y_{r, j}= \begin{cases}\overline{x_{r, j}} & \text { if } j=l-2(r-1)+1, \cdots, l-1 \\ x_{r, j} & \text { otherwise }\end{cases}
$$

for $j=2,4, \cdots, 2(n-r)$.

By (4.7) and (4.8), we have

$$
y_{r, j}= \begin{cases}\overline{x_{r, j}} & \text { if } j=l-2(r-1), \cdots, l \\ x_{r, j} & \text { othervise }\end{cases}
$$


which shows that (4.2) is valid for the $r$-th row when it does hold for the $(r-1)$-th row for $1 \leqq r \leqq(l-1) / 2$.

The similar method can be applied to prove that (4.2) is valid for $r>(l-1) / 2$. This gives the proof of the validity of the assertion of (4.2) for odd $l$.

The construction of $\mathrm{C}_{(1, l)}(X)$ for even integer $l$ and that of $\mathrm{C}_{(k, 2)}(X)$ for $k=2,3$, $\cdots, n-1$ can be similarly done. This completes the proof of LEMMA 4.

In our cell space approach, a state of each cell in $\Delta^{(n)}$ cell space can take either 1 or 0 . Let us introduce a notion of state variable of a cell which represents a state of the cell and hence can take either 1 or 0 . Now our definition of determinative subspace implies that this is a set of $3(n-1)$ cells each of which state can be assigned mutually independently by which the state of all remaining cells are uniquely determined. A determinative subspace implies and is implied by a set of such $3(n-1)$ mutually independent state variable each of which represents a state of each cell belonging respectively to its determinative subspace and hence can take either 1 or 0 .

COROLlARY 1. There exists one to one correspondence between a state of each cell $(i, j)$ in $\mathrm{D}_{0}^{(n)}$ and that of arbitrary one cell in $\mathrm{E}_{0}^{(n)}(i, j)$, if the state of all the remaining cells in $\mathrm{D}_{0}^{(n)}$ are fixed. In the consequence any one cell in $\mathrm{E}_{0}^{(n)}(i, j)$ can take place of the cell $(i, j)$ in $\mathrm{D}_{0}^{(n)}$ in order to be determinative subspace.

In view of LEMMA 4 and COROLlaRy 1 , we observe

THEOREM 2. Let $(i, j)$ be any assigned cell in $\mathrm{D}_{0}^{(n)}$ and let us write $\mathrm{D}_{0}^{(n)}-(i, j)=\mathrm{S}_{i j}$. Then we have

(a) a set of cells consisting of $\mathrm{S}_{i j}$ and one arbitrary cell in $\mathrm{E}_{0}^{(n)}(i, j)$ is a determinative subspace in the $A^{(n)}$ cell space.

(b) a set of cells consisting of $\mathrm{S}_{i j}$ and one arbitrary cell in $\mathrm{E}_{0}^{(n)}(i, j)-\mathrm{G}_{\mathrm{S}_{i j}}(i, j)$ is a non-generative determinative subspace in the $J^{(n)}$ cell space.

It will be sufficient to notice that the latter assertion (b) is due to the assertion (a) and the notion of the same generation with the cell $(i, j)$ with reference to $S_{i, j}$. By removing the set $\mathrm{G}_{s_{i}, j}(i, j)$ from $\mathrm{E}_{0}^{(n)}(i, j)$, our determinative subspace given in (a) become NGDS.

[2] SupPerposition OF ElEMENTARy SUbSETS AND DECOMPOSITION OF THE WHOLE CELL SPACE INTO A FAMILY OF SUPERPOSED ELEMENTARY SUBSETS

For the sake of simplicity, let us denote for a moment

and

$$
i \equiv(1, i) \quad \text { for } i=i, 2,3, \cdots, 2 n-1 \text {, }
$$

$$
i \equiv(j, 2) \quad \text { for } i=2 n+(j-2), j=2,3, \cdots, n-1 \text {. }
$$

Let us consider a set of $m$ different cells arbitrarily chosen from $\mathrm{D}_{0}^{(n)}$, which we shall denote by $\left\{i_{\alpha} ; \alpha=1,2, \cdots, m\right\}$. For the sake of simplicity, let us denote

$$
\begin{aligned}
& \mathrm{A}_{i_{\alpha}}^{1} \equiv \mathrm{E}_{0}^{(n)}\left(i_{\alpha}\right) \\
& \mathrm{A}_{i_{\alpha}}^{0} \equiv \mathrm{A}_{i_{\alpha}}^{1} \quad \text { (complement of } \mathrm{A}_{i_{\alpha}}^{1} \text { in } \Delta^{(n)} \text { ) }
\end{aligned}
$$

for $\alpha=1,2, \cdots, m$.

The $2^{m}$ sets of cells defined by 


$$
\left\{\mathrm{A}_{i_{1}}^{\varepsilon_{1} \mathrm{i}_{1}} \mathrm{~A}_{i_{2}}^{\left.\varepsilon_{i_{2}} \cdots \mathrm{A}_{i m}^{\varepsilon_{i}}\right\}},\right.
$$

where $\varepsilon_{i_{\alpha}}=1$ or 0 for $\alpha=1 \sim m$, give us a division of the whole $\Delta^{(n)}$ space into a family of mutually disjoint sets. This decomposition is called to be a decomposition of the whole $J^{(n)}$ space into a family of superposed elementary subsets. An introduction of the notation of superposition may be justified by the fact that the state variable correspondending to any cell belonging to a set $\mathrm{A}_{i_{1}{ }_{1}}^{\varepsilon_{1}} \mathrm{~A}_{i_{2}}^{\varepsilon i_{2}} \cdots \mathrm{A}_{i_{m}}^{\varepsilon_{i m}}$ can be expressed by the Boolean sum of the form

$$
\varepsilon_{i_{1}} \zeta_{i_{1}}+\varepsilon_{i_{2}} \zeta_{i_{2}}+\cdots+\varepsilon_{i_{m}} \zeta_{i_{m}},
$$

where each $\zeta_{i_{r}}(\alpha=1,2, \cdots, m)$ denotes the state variable corresponding to the cell $i_{\alpha}$.

Particularly when $m$ is equal to the size $3(n-1)$ of determinative subspace, then the expression (4.11) amounts to assert that for any assigned cell $(k, l)$ in the $\Delta^{(n)}$ cell space there corresponds one and only one set of $\left\{\varepsilon_{\alpha}^{(k, t)}(\alpha=1,2, \cdots, 3(n-1))\right\}$ where $\varepsilon_{\alpha}^{(k, l)}=1$ or 0 , such that the state variable $\eta_{k, l}$ corresponding to the cell $(k, l)$ is denote by

$$
\eta_{k, l}=\varepsilon_{1}^{(k, l)} \zeta_{1}+\varepsilon_{2}^{(k, l)} \zeta_{2}+\cdots+\varepsilon_{u}^{(k, l)} \zeta_{u}
$$

where $u=3(n-1)$.

For any set $\mathrm{S}$ of $m$ cells $\left(k_{,}, l_{\hat{\beta}}\right)(\beta=1,2, \cdots, m)$ in $\Delta^{(n)}$ cell space there corresponds a matrix

$$
M_{D_{0}^{(n)}}(S)=\left(\begin{array}{cccc}
\varepsilon_{1}^{\left(k_{1}, l_{1}\right)} & \varepsilon_{2}^{\left(k_{1}, l_{1}\right)} & \cdots & \varepsilon_{u}^{\left(k_{1}, l_{1}\right)} \\
\varepsilon_{1}^{\left(k_{2}, l_{2}\right)} & \varepsilon_{2}^{\left(k_{2}, l_{2}\right)} & \cdots & \varepsilon_{u}^{\left(k_{2}, l_{2}\right)} \\
\cdots & \ldots \ldots \ldots \ldots \ldots \ldots \ldots . . . \cdots \\
\varepsilon_{1}^{\left(k_{m}, l_{m}\right)} \varepsilon_{2}^{\left(k_{m}, l_{m}\right)} & \cdots & \varepsilon_{u}^{\left(k_{m}, l_{m}\right)}
\end{array}\right)
$$

Definition 13. A set $\mathrm{F}$ of $3(n-1)$ cells in a $\Delta^{(n)}$ cell space is called to be an $m$-replacement deformation set of $\mathrm{D}_{0}^{(n)}$ if $\mathrm{F}$ is obtained by the following two procedures :

(1) Addition of a set $\mathrm{A}$ of $m$ cells which belong to the set $\Delta^{(n)}-\mathrm{D}_{0}^{(n)}$

(2) Removal of a set $\mathrm{R}$ of $m$ cell belonging to $\mathrm{D}_{0}^{(n)}$.

DEFINITION 13 implies that the necessary and sufficient condition that $F$ is an $m$-replacement deformation set of $\mathrm{D}_{0}^{(n)}$ if and only if there exists two set $\mathrm{A}$ and $\mathrm{R}$ such that

(i) $\mathrm{R}=\left\{i_{\alpha} \in \mathrm{D}_{0}^{(n)}, \alpha=1,2, \cdots, m\right\}$

(ii) $\mathrm{A}=\left\{\left(k_{\beta}, l_{\beta}\right) \in \Delta^{(n)}-\mathrm{D}_{0}^{(n)}, \beta=1,2, \cdots, m\right\}$

(iii) $\mathrm{F}=\mathrm{D}_{0}^{(n)}+\mathrm{A}-\mathrm{R}$.

Now let us enunciate the following

THEOREM 3. The necessary and sufficient condition that an m-replacement deformation set $\mathrm{F}$ of the determinative subspace $\mathrm{D}_{0}^{(n)}$ is a determinative subspace is that the rank of $\mathrm{M}_{\mathrm{D}_{0}}(n)$ (A) is equal to $m$.

PROOF. (a) The sufficiency Let the rank of $\mathrm{M}_{\mathrm{D}_{0}}(n)$ (A) be $m$. Then we can find a set $m$ positive integer $\left\{i_{\alpha}, \alpha=1,2, \cdots, m\right), 1 \leqq i_{1}<i_{2}<\cdots<i_{m} \leqq 3(n-1)$ such that the $m \times m$ determinant 
has the rank $m$.

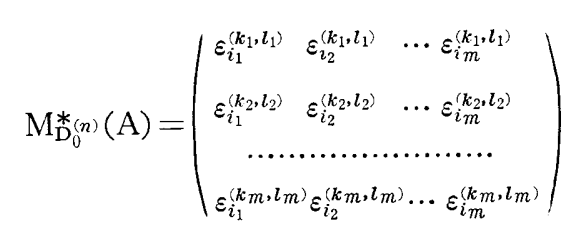

In view of this fact there does exist one to one correspondence between a configuration in $\mathrm{A}$ and that in $\mathrm{R}$ where $\mathrm{R}$ is defined as the set $\left\{i_{\alpha}\right\} \quad(\alpha=1,2, \cdots, m)$ in $\mathrm{D}_{0}^{(n)}$. This implies that $\mathrm{F}$ is a determinative subspace.

(b) The necessary Let $\mathrm{F}$ be an $m$-replacement deformation set of $\mathrm{D}_{0}^{(n)}$ and let it be a determinative subspace. Since both of the two subsets $D_{0}^{(n)}=\left(D_{0}^{(n)}-R\right)+R$ and $F=\left(D_{0}^{(n)}-R\right)+A$ are determinative with the common component $\left(D_{0}^{(n)}-R\right)$, there exists one to one correspondence between a configuration in $R$ and that in $A$ for any assigned configuration in $\mathrm{D}_{0}^{(n)}-\mathrm{R}$. If the rank of $\mathrm{A}$ would be less than $m$, then it is impossible to have such a one to one correspondence. Since the rank A is not greater than $m$, as we were to prove.

Now we shall give the following Theorem concerned with NGDS.

THEOREM 4. The necessary and sufficient condition that an m-replacement deformation set $\mathrm{F}$ of the determinative subspace $\mathrm{D}_{0}^{(n)}$ is a non-generative determinative subspace (NGDS) is that there exists at least one $\left(k_{\beta}, l_{\beta}\right)$ in A such that the cell $\left(k_{\beta}, l_{\beta}\right)$ does not belong to the set $\left\{\mathrm{G}_{\mathbf{D}_{0}^{(n)}-i_{n}} ; \alpha=1,2, \cdots, m\right\}$ of generations of all cells in $\mathrm{R}$ and the rank of $\mathrm{M}_{\mathrm{D}_{0}^{(n)}(\mathrm{A})}$ is equal to $m$.

PROOF. It will be sufficient to notice that the assertion of THEOREM 4 is due to the assertion of THEOREM 3 and the notion of the generation with the cell $i_{\alpha}$ with reference to $\mathrm{D}_{0}^{(n)}-i_{\alpha}$. By removing the set of all generations $\left\{\mathrm{G}_{\mathrm{D}_{0}^{(n)}-i_{\alpha}} ; \alpha=1,2, \cdots, m\right\}$ from $A$, our determinative subspace given in THEOREM 3 become NGDS.

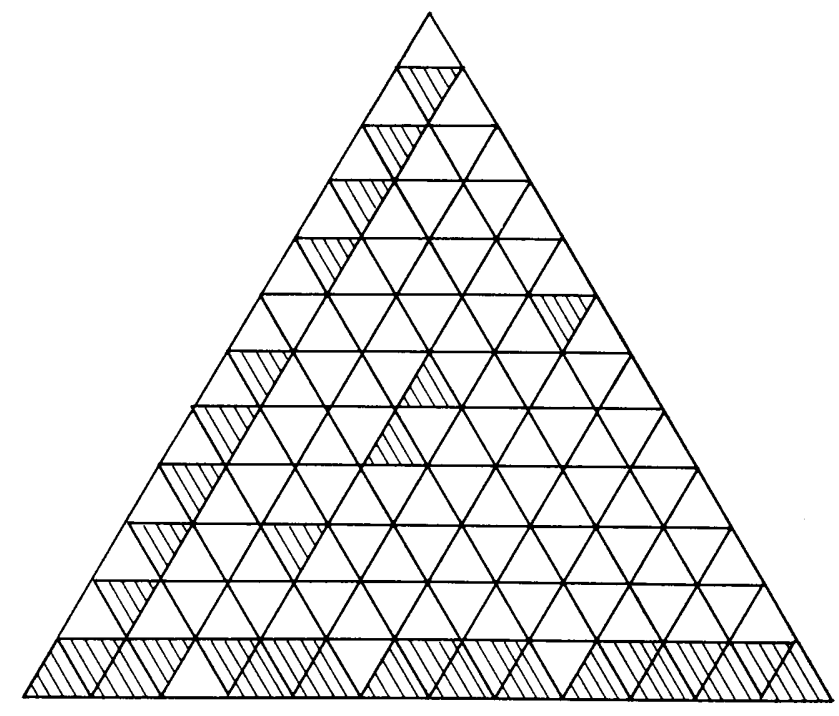

Fig. 4.1. NGDS of $\Delta^{(12)}$ cellspece. 
By this THEOREM 4, we can construct any NGDS in $4^{(n)}$ cell space. Let us show an example of NGDS in $4^{(12)}$ cell space in Figure 4.1.

\section{$\S 5$. Structure of stable configuration in $J^{(n)}$ cell space}

The purpose of this SECTION is to give the other proof of THEOREM 2 in our paper Yamaguchi [2] by using our fundamental Lemma 4 in SECTION 4.

THEOREM 5. Any stable configuration $\mathrm{C}(X)$ in a cell space can be expressed as a supperposition of the elementary stable configurations to the effect that

$$
\mathrm{C}(X)=\sum_{j=1}^{2 n-1} x_{i, j} \mathrm{C}\left(\mathrm{E}_{0}^{(n)}(1, j)\right)+\sum_{i=2}^{n-2} x_{i, j} \mathrm{C}\left(\mathrm{E}_{0}^{(n)}(i, 2)\right),
$$

where the additions in the right hand side are given according to Boolean algebra. Conversely, for each assigned configuration $\mathrm{D}_{0}^{(n)}(X)$ in $\mathrm{D}_{0}^{(n)}$, the right hand side of (5.1) gives us a stable configuration in $\Delta^{(n)}$ cell space.

Proof. We shall first show that any stable configuration $\mathrm{C}(X)$ in $\Delta^{(n)}$ cell space can be expressed as (5.1). Let us denote by $\left\{\left(1, j_{1}\right),\left(1, j_{2}\right), \cdots,\left(1, j_{l}\right),\left(i_{1}, 2\right),\left(i_{2}, 2\right), \cdots\right.$, $\left.\left(i_{k}, 2\right)\right\}(0 \leqq l \leqq 2 n-1,0 \leqq k \leqq n-1)$ the set of all cells in $\mathrm{D}_{0}^{(n)}$ each of whose state is one in the stable configuration $C(X)$. Then (5.1) is equivalent to the relation

$$
\mathrm{C}(X)=\sum_{s=1}^{\iota} \mathrm{C}\left(\mathrm{E}_{0}^{(n)}\left(1, j_{s}\right)\right)+\sum_{r=1}^{k} \mathrm{C}\left(\mathrm{E}_{0}^{(n)}\left(i_{r}, 2\right)\right) \text {. }
$$

Let us denote by $\mathrm{D}_{0}^{(n)}\left(X ;\left(1, u_{1}\right),\left(1, u_{2}\right), \cdots,\left(1, u_{p}\right),\left(v_{1}, 2\right), \cdots,\left(v_{q}, 2\right)\right)$ the configuration in $\mathrm{D}_{0}^{(n)}$ such that ths state of each cell in set $\left(\left(1, u_{1}\right),\left(1, u_{2}\right), \cdots,\left(1, u_{p}\right),\left(v_{1}, 2\right)\right.$, $\left.\left(v_{2}, 2\right), \cdots,\left(v_{q}, 2\right)\right)$ is one and the state of each other cell in $\mathrm{D}_{0}^{(n)}$ is zero, and by $\mathrm{C}(X$; $\left.\left(1, u_{1}\right),\left(1, u_{2}\right), \cdots,\left(1, u_{p}\right),\left(v_{1}, 2\right),\left(v_{2}, 2\right), \cdots,\left(v_{q}, 2\right)\right)$ the stable configuration determined by $\mathrm{D}_{0}^{(n)}\left(X ;\left(1, u_{1}\right),\left(1, u_{2}\right), \cdots,\left(1, u_{p}\right),\left(v_{1}, 2\right),\left(v_{2}, 2\right), \cdots,\left(v_{q}, 2\right)\right)$. By LemmA 4 we have

$$
\begin{aligned}
& \mathrm{C}\left(X ;\left(1, j_{1}\right)\right)=\mathrm{O}^{(n)}+\mathrm{C}\left(\mathrm{E}_{0}^{(n)}\left(1, j_{1}\right)\right)=\mathrm{C}\left(\mathrm{E}_{0}^{(n)}\left(1, j_{1}\right)\right) \\
& \mathrm{C}\left(X ;\left(1, j_{1}\right),\left(1, j_{2}\right), \cdots,\left(1, j_{s}\right)\right)=\mathrm{C}\left(X ;\left(1, j_{1}\right), \cdots,\left(1, j_{s-1}\right)\right)+\mathrm{C}\left(\mathrm{E}_{0}^{(n)}\left(1, j_{s}\right)\right),
\end{aligned}
$$

for $s=2,3, \cdots, l$,

$$
\begin{aligned}
& \mathrm{C}\left(X ;\left(1, j_{1}\right), \cdots,\left(1, j_{l}\right),\left(i_{1}, 2\right), \cdots,\left(i_{r}, 2\right)\right) \\
& \quad=\mathrm{C}\left(X ;\left(1, j_{1}\right), \cdots,(1, j),\left(i_{1}, 2\right), \cdots,\left(i_{r-1}, 2\right)\right)+\mathrm{C}\left(\mathrm{E}_{0}^{(n)}\left(i_{r}, 2\right),\right.
\end{aligned}
$$

for $r=2,3, \cdots, k$.

The repeated applications (5.3), (5.4) and (5.5) yields us

$$
\begin{aligned}
\mathrm{C}(X) & =\mathrm{C}\left(X ;\left(1, j_{1}\right), \cdots,\left(1, j_{l}\right) \cdot\left(i_{1}, 2\right), \cdots,\left(i_{k}, 2\right)\right) \\
& =\sum_{s=1}^{\ell} \mathrm{C}\left(\mathrm{E}_{0}^{(n)}\left(1, j_{s}\right)\right)+\sum_{r=1}^{k} \mathrm{C}\left(\mathrm{E}_{0}^{(n)}\left(i_{r}, 2\right)\right) .
\end{aligned}
$$

Therefore any stable configuration $\mathrm{C}(X)$ can be expressed as (5.1).

It is clear that the right hand side of (5.2) gives us a stable configuration in the $\Delta^{(n)}$ cell space, because the sum of states of any basic cell space in the stable configuration $\mathrm{C}\left(\mathrm{E}_{0}^{(n)}\left(1, j_{s}\right)\right)(s=1,2, \cdots, l)$ and $\mathrm{C}\left(\mathrm{E}_{0}^{(n)}\left(i_{r}, 2\right)\right)(r=1,2, \cdots, k)$ is zero modulo 2 . 


\section{§6. Acknowledgement}

In conclusion of this paper the author wishes to express the hearty thanks to Proffessor Tosio Kitagawa for his constant encouragements and his kind advices during the preparation of this work.

[Appendix] An example of our construction process of generative determinative subspace given in Section 3

We shall give an example of our construction process of GDS in $\Delta^{(6)}$ cell space in Figure 1. The determinative subspace $\mathrm{D}_{i}(i=1,2, \cdots, 13)$ is given by the set of cell with hatches and the subset $S_{i}(i=1,2, \cdots, 13)$ determined by $D_{i}$ is given by the two sets of cells with hatches and spots in Figure 1.

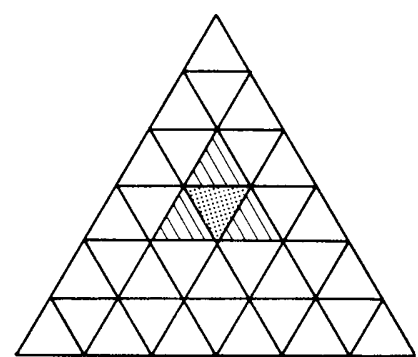

$D_{1}$ and $S_{1}$

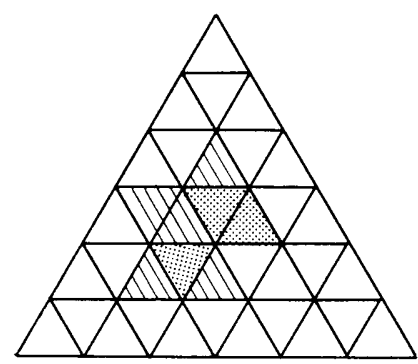

$\mathrm{D}_{3}$ and $\mathrm{S}_{3}$

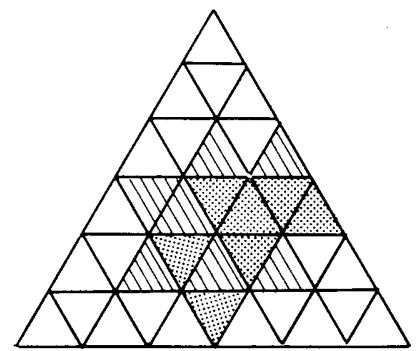

$\mathrm{D}_{5}$ and $\mathrm{S}_{5}$

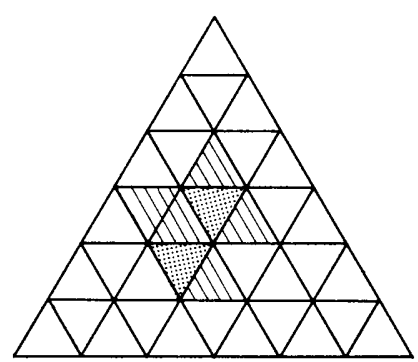

$\mathrm{D}_{2}$ and $\mathrm{S}_{2}$

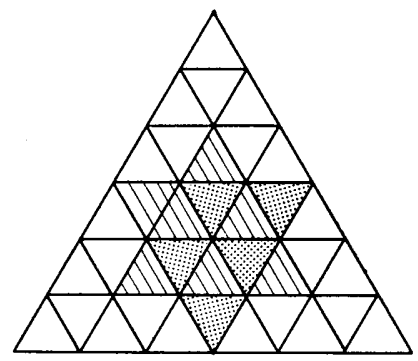

$\mathrm{D}_{4}$ and $\mathrm{S}_{4}$

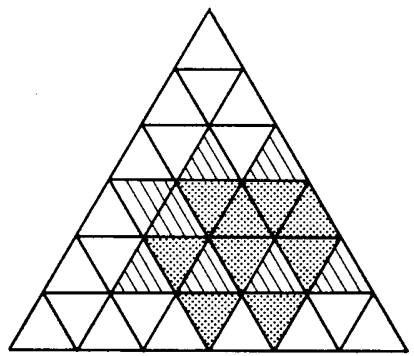

$D_{6}$ and $S_{6}$ 

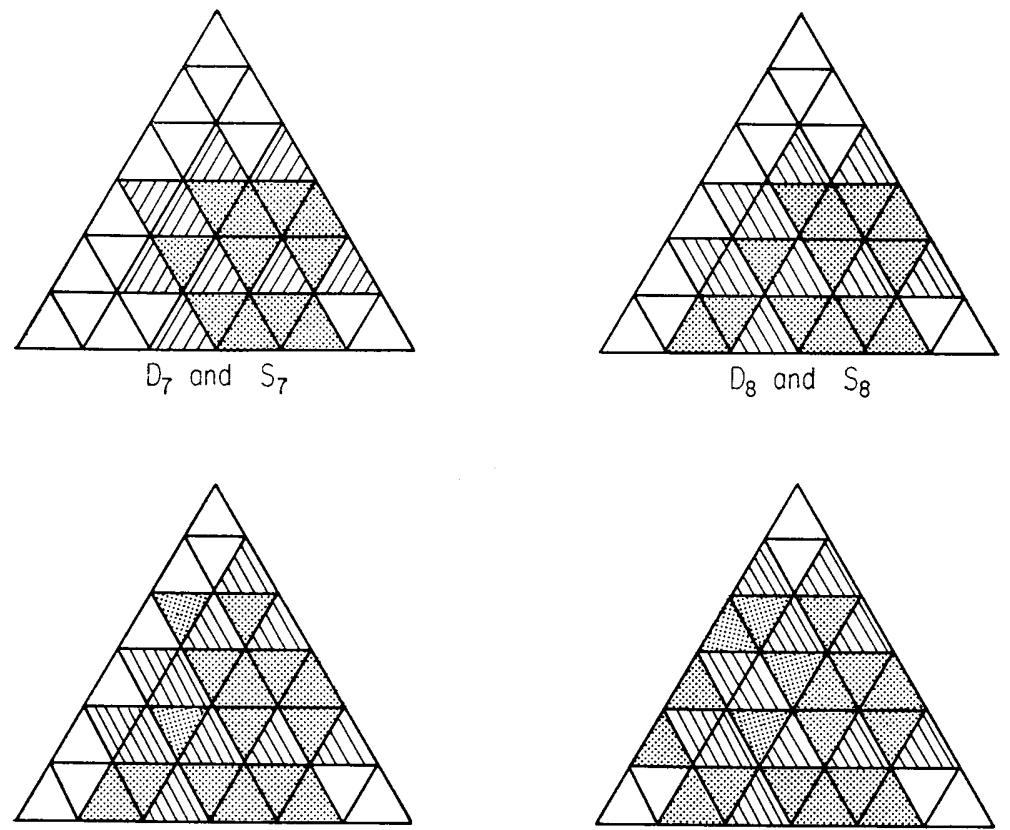

$\mathrm{D}_{9}$ and $\mathrm{S}_{9}$

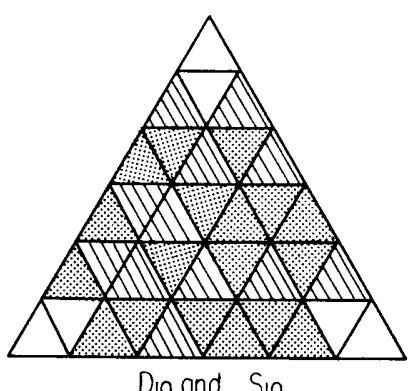

$D_{10}$ and $S_{10}$

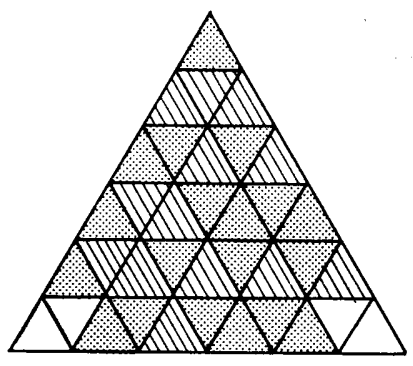

$D_{11}$ and $S_{11}$

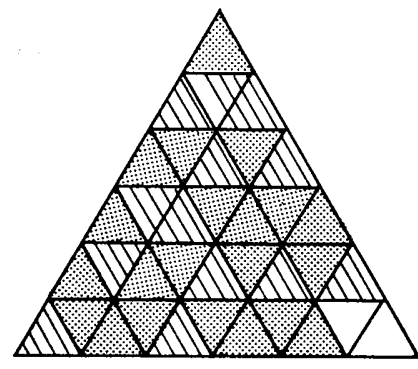

$D_{12}$ and $S_{12}$

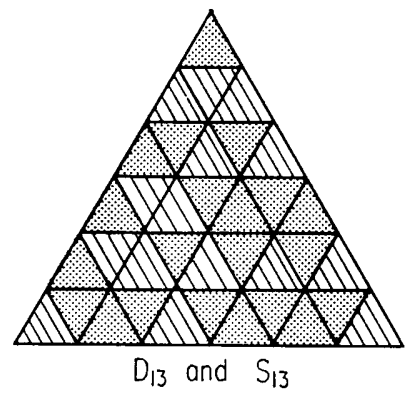

Figure 1. Construction process of GDS in $\Delta^{(6)}$ cell space. 


\section{Literature}

[1] Kitagawa, T. and Yamaglchi, M.: Local Majority Transformation in Cell SpaceInformation Science Approach to Biomathematics, II, Research Report of the Research: Institute of the Fundamental Information Science, Fac. Sci. Kyushu Univ., No. 10, 1970. Bull. Math. Statist., Vol. 14, No. 3/4 (1971), 61-82.

[2] Yamaguchi, M.: Stable Configuration under Local Majority Transformations on Cell Space-Information Science Approach to Biomathematics, V, Research Report of the Research Institute of the Funnamental Information Science, Fac. Sci. Kyushu Univ., No. 13, 1970. Bull. Math. Statist., Vol. 14, No. 3/4 (1971), 93-106.

[3] Kitagawa, T. and Yamaguchi, M.: Determinative Subspace for Stable Configuration under Local Majority Transformations on Cell Space-Information Science Approach to. Biomathematics, VI, Research Report of the Research Institute of the Fundamental Information Science, Fac. Sci. Kyushu Univ., No. 15, 1970.

[4] Kitagawa, T.: The Second Prolegomena to Cell Space Approaches-Information Science Approach to Biomathematics, VII, Research Report of the Research Institute of the: Fundamental Information Science, Fac. Sci. Kyushu Univ., No. 16, 1970.

\section{ERRATA}

of

\section{“On a Statistical Analysis of Homogeneous Random Fields on a Metric Space acted upon by a Compact Metric Group".}

Bulletin of Mathematical Statistics, Vol. 13, No. 3-4 (1969) pp. 65-92.

By

\section{Takeaki NAGAI}

(Received December 14, 1970)

In Example 2.1, p. 69, of $\S 2$, the disk $A$ and the set $V$ should by replaced by a subset $\Lambda^{\prime}=\Lambda-S_{\varepsilon}(0)$ of $\Lambda$ where $S_{\varepsilon}(0)=\left\{(x, y) \mid x^{2}+y^{2}<\varepsilon\right\}, 0<\varepsilon<r$, and by a $V^{\prime}$ $=\{(x, 0) \mid \varepsilon \leqq x \leqq r\}$.

However, this cause no essential damage in example 2.1, since $\varepsilon>0$ can be taken an arbitrarily small and we can take the measure $\mu\left(S_{\varepsilon}(0)\right)$ as small as possible.

Example 2.2, P. 69 , of $\S 2$, should be removed, since the space $L_{3}$ and the group G in the example 2.2 does not satisfy the conditions stated in earlier part of $\S 2$. The removal of the example 2.2, however, does not affect theorem 2.1 and the ensuing discussions. 